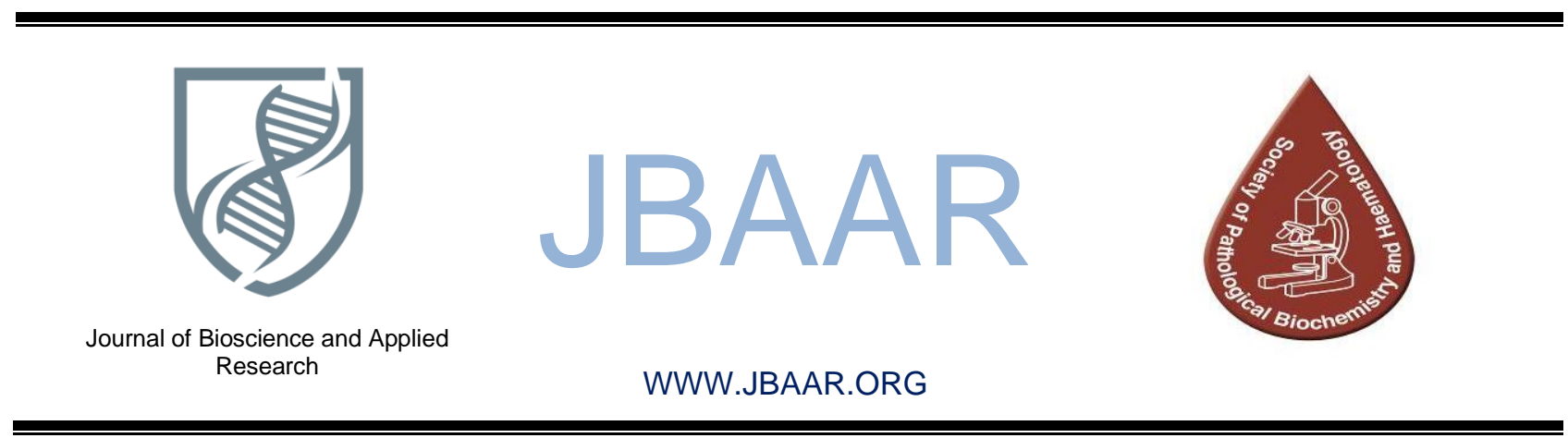

\title{
Investigating the effect of therapeutic ultrasound irradiation on the liver and kidney function of male albino mice
}

\author{
Moustafa Hussein Moustafa \\ "Biophysics Department, Medical research institute, Alexandria University Egypt \\ DOI: $10.21608 /$ jbaar.2017.124880
}

\begin{abstract}
In the current explanatory study, experimental methods were conducted to anticipate the effect of therapeutic ultrasound irradiation on the liver and kidney of male albino mice. The histopathological studies revealed a convenient apparent aberration. These findings were supported by hematological investigation of complete blood count (CBC), blood enzymes like aspartate aminotransferase (AST or SGOT) and alanine aminotransferase (ALT or SGPT), also Creatinine and Urea levels were detected. The results showed no significant alteration in the physiological function of both liver and kidney. The level of malondialdehyde (MDA) and total antioxidant contents were carried out, the level of Glutathione (GST), Superoxide dismutase (SOD) was still in normal ranges.
\end{abstract}

Keywords: Non-ionizing radiation, ultrasound, free radicals, antioxidants.

\section{Introduction}

Nowadays, Cutting-edge technology increases the exposure rate of electromagnetic waves (EM) irradiation. The daily lifestyle sources may be industrial noising, cell phones, and computers, home-aid tools like microwave, oven, and electric heater. Consequently, great attention has been paid to the effects of electromagnetic radiation on a living organism ${ }^{1}$.

EM includes ionizing (such accelerated atomic particles, infrared laser) and non-ionizing radiations (high-intensity ultrasound). NonIonizing radiations have sufficient energy only for excitation, instead of producing charged ions when passing through matter like ionizing ${ }^{\mathbf{2}, 3}$.
Ultrasound waves are widely used in ordinary lifestyle Industry exposure (ultrasonic washers, welding and erosion machines), consumer devices (burglaralarms), dog whistles, bird and rodent repellents, humidifiers, and inhalers. These types of US waves are of a low frequency of an average of $20 \mathrm{kHz}$. While higher frequency (over $0.8 \mathrm{MHz}$ ) is used in medical therapeutic and diagnostic purposes $^{1-3}$.

The biologic effects of ultrasound can be thermal and non-thermal. Although most of the biological reactions are due to the thermal effect, the nonthermal effects of ultrasound include, e.g., cell membrane gaseous cavitations ${ }^{4}$.

Radiation exposure, especially ionizing one, may result in the liberation of oxygen and nitrogen reactive species known as free radicals within the 
cells ${ }^{5}$.these radicals have unpaired electrons making them highly reactive species ${ }^{6}$. In mammals, a sophisticated system of antioxidants is produced within the liver to counteract the action of these reactive species. Catalase, Superoxide dismutase, and glutathione peroxidase are enzymatic A lot of risk factors, including alcohol, drugs, environmental pollutants, and irradiation, may induce oxidative stress in the liver, which in turn results in severe liver diseasesError! Bookmark not defined. ${ }^{6}$. The current explanatory study and experimental methods are conducted to anticipate the effect of therapeutic ultrasound irradiation on the liver and kidney of male albino mice. The study protocol will be conducted at a cellular level.

\section{Material and methods}

\section{Ultrasonic unit}

An ultrasonic therapy instrument was used (Model CS1 Shanghai, No. 822 Factory. China). It operates at a frequency of $0.8 \mathrm{MHz}$ and power output is converted to ultrasonic mechanical energy using an ultrasonic transducer (calcium zirconate -titanate). The mechanical ultrasonic energy has a beam power density which can be adjusted from 0.5 to $3 \mathrm{~W} / \mathrm{cm}^{2}$. Sonication time can be adjusted up to 30 minutes, while the set-time is over, the power supply is cut off automatically and intermittent alarming sound may be given. This instrument operates at both continuous wave mode with output power from $0.5-3 \mathrm{~W} / \mathrm{cm}^{2}$ adjustable in 11 steps and pulsed mode (pulse frequency $1000 \mathrm{~Hz}$, duty ratio $1 / 3$, and average power density from $0.15-1$ $\left.\mathrm{W} / \mathrm{cm}^{2}\right)$.

\section{Methods}

Sample groups: The mice sample size was 15 albino mice were divided into groups as shown below:

\section{- Group I: 5mice non radiated act as a control group.}

- Group III: 10 mice, ultrasound group as follow:

a) 5 mice: We're exposed to pulsed ultrasound at a power density of $3 \mathrm{~W} / \mathrm{cm}^{2}$ for 3 minutes. examples of antioxidants, while Glutathione, vitamin $\mathrm{C}$ is a nonenzymatic electron receptor antioxidant ${ }^{7}$. Lipid peroxidation is a consequence of oxidative stress. Malondialdehyde is the major indicator of lipids peroxidation rate . $^{8}$.

b) 5 mice: We're exposed to continuous ultrasound at a power density of $3 \mathrm{~W} / \mathrm{cm}^{2}$ for 3 minutes.

\section{Histopathological Examination}

Small pieces of freshly excised organs of (liver, and kidney) of all the experimental groups were processed and examined by Hematoxylin and Eosin (H\&E) method as follows: The small pieces of organs were fixed at $10 \%$ formaldehyde. Dehydration in ascending grades using alcohol was performed. Paraffin blocks were performed embedded in paraffin. Then Clean with xylene followed by rehydration in descending grades of alcohol. Stain the samples with Hematoxylin and Eosin stain, then Cleaned again with ethylene. Finally, the slides were prepared to be examined by light microscopy

\section{Haematological and serological study}

The protocol of blood collection for hematological studies was quoted from $M$ Salahudd et $a l^{9}$. The collected blood was divided into three portions. About $2 \mathrm{ml}$ portion was taken in evacuated EDTAcontaining tube for hematological studies on the same day. The remaining blood was used for the collection in a plain tube for serological studies. The serum was separated and centrifuged to remove unwanted blood cells where necessary.

The samples were stored at $-20^{\circ} \mathrm{C}$. Liver enzymes such" Alanine aminotransferase, GPT, and serum albumin were assayed. Kidney function was assayed by conventional "urea, creatinine and total protein contents". using a Hitachi 911 automated analyzer using spectrum Kits according to the manufacturer's specifications.

Statistical analysis: The data were analyzed statistically between normal and treated values by one way ANOVA with post-hoc Duncan's multiple range test.

\section{Biochemical and serological study}




\section{Estimation of Serum aspartate transaminase (AST/GOT) and AlanineTransaminase $(\text { ALT/POT })^{10}$}

ALT enzyme is highly concentrated in the liver and lower extent in kidney and heart muscles, pancreas, and lungs. It can be elevated in a case like Hepatitis, cirrhosis, obstructive jaundice, liver carcinoma. Meanwhile, the AST enzyme concentrates mainly in the heart, heart, liver, muscles, and kidney. Although both enzymes are elevated whenever liver cells are affected, ALT is the liver-specific one. The assay principle of both enzymes is the same. ALT activity is monitored by the concentration of pyruvate hydrazone, formed with 2,4-dinitrophenylhydrazine. While in AST by concentration oxaloacetatehydrazone.

\section{Alkaline phosphatase}

Alkaline phosphatase was estimated by the Kinetic method according to the recommended reference method of DGKC. Liquid stable double reagent. (ALP) hydrolyzes the colorless p-nitrophenyl phosphate to p-nitrophenol and phosphate in the presence of magnesium ions. The product of enzyme hydrolysis p-nitrophenol has a yellow color at the $\mathrm{pH}$ of the reaction. The working procedure was followed according to determination kits.

\section{Creatinine and Urea levels ${ }^{11}$}

Creatine is a metabolite synthesized in the kidney, liver, and pancreas. It is transported to other organs such as muscle and brain where it is phosphorylated to phosphocreatine. Some free creatines are converted to creatinine in muscle cells daily. Urea is the main end product of protein nitrogen metabolism. It is synthesized throughout the urea cycle from ammonia in liver cells. Both serum creatinine and urea levels are elevated in renal malfunction, especially decreased glomerular filtration. Unlike creatinine serum urea levels may be affected by dehydration, diet, and protein metabolism. Thus serum creatinine is a significantly more reliable renal function screening test than serum urea.
Creatinine is monitored by a colored complex formed by the reaction of creatinine reacts with picric acid in an alkaline solution. While urea is hydrolyzed to ammonia a carbon dioxide by urease in alkaline media, a colored complex is formed in the presence of an indicator which is proportional to urea concentration.

\section{Estimation of serum Malondialdehyde (MDA)}

The procedure was adopted from Deepa D'souza et al 8 as follows; $2 \mathrm{~mL}$ of blood was collected. The serum was separated by centrifuging the blood sample at $3000 \mathrm{rpm}$ for $5 \mathrm{~min}$. Following which the serum MDA was measured using the method of Buege (1978). Serum-100 $\mu \mathrm{L}$ serum is diluted to $500 \mu \mathrm{L}$ with distilled water. The samples are kept in a boiling water bath for $15 \mathrm{~min}$. To the diluted sample $1 \mathrm{~mL}$ of Trichloroacetic acid TCA-2thiobarbituric acid (TBA)- $\mathrm{HCl}$ reagent is added. The reaction mixture is cooled and centrifuged. The supernatant is taken and the optical density of the pink color formed is read at $535 \mathrm{~nm}$. The concentration of MDA in the sample is got by plotting the obtained absorbance against the standard graph. The optical density of the pink color formed is directly proportional to the concentration of serum MDA in the given sample. Sample concentration is calculated from the following: sample $=\mathrm{A}$ sample $\div$ A standard $\times 10$ $\mathrm{nmol} / \mathrm{ml}$

\section{Estimation of plasma total antioxidant capacity}

The determination of the antioxidative capacity is performed by the reaction of antioxidants in the sample with a defined amount of exogenously provide hydrogen peroxide $\left(\mathrm{H}_{2} \mathrm{O}_{2}\right)$. The antioxidants in the sample eliminate a certain amount of the provided hydrogen peroxide. The residual $\mathrm{H}_{2} \mathrm{O}_{2}$ is determined colorimetrically by an enzymatic reaction which involves the conversion of 3,5, dichloro-2-hydroxy benzenesulfonate to a colored product. Total antioxidant Sample concentration is calculated from the following $=$ absorbance blank-sample absorbance $\times 3.33 \quad(\mathrm{~m}$ $\mathrm{M} / \mathrm{L}$ ) 


\section{Superoxide dismutase (SOD)}

Colorimetric that produces a water-soluble formazan dye upon reduction with superoxide anion. The rate of the reduction with a superoxide anion is linearly related to the xanthine oxidase activity and is inhibited by SOD. Therefore, the inhibition activity of SOD can be determined by a colorimetric method.

\section{Glutathione reductase (GR)}

GR reduces GSSG to GSH, which reacts with 5, 5'-dithiobis (2-nitrobenzoic acid) (DTNB) to generate TNB2- (yellow color, ? $\max =405 \mathrm{~nm}$ ).

\section{Catalase}

It catalyzes the decomposition of hydrogen peroxide $\left(\mathrm{H}_{2} \mathrm{O}_{2}\right)$ to water and oxygen. Catalase is a

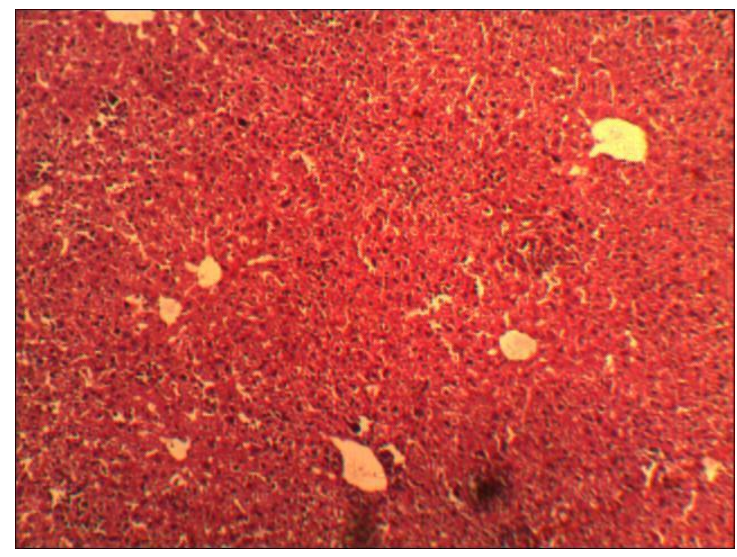

\section{A: LIVER}

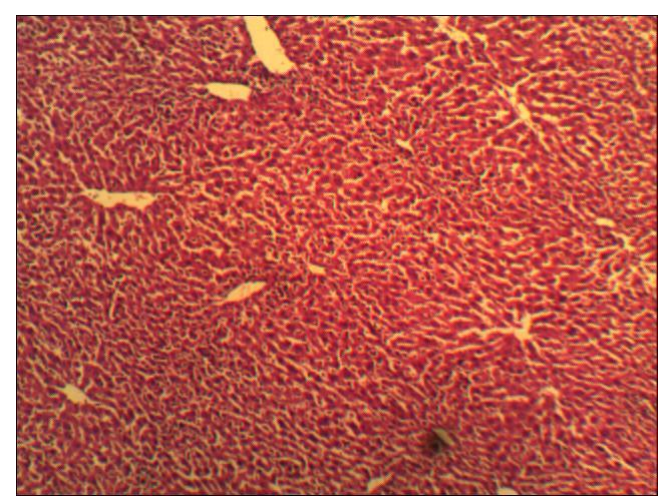

B: US irradiated Liver tetramer of four polypeptide chains and contains four porphyrin heme (iron) groups that allow the enzyme to react with hydrogen peroxide. The optimum $\mathrm{pH}$ for human catalase is approximately $\mathrm{pH} 7$, with a fairly broad maximum as the rate of reaction does not change appreciably between $\mathrm{pH}=$ 6.8-7.5.

\section{Results and discussion}

The macroscopic photographs were captured to investigated histopathological integrity for both liver and kidney of irradiated samples relative to the control group. The microscopic photos in Figure 1 show no aberrant alteration in the cellular integrity for both liver and kidney samples compared with control.

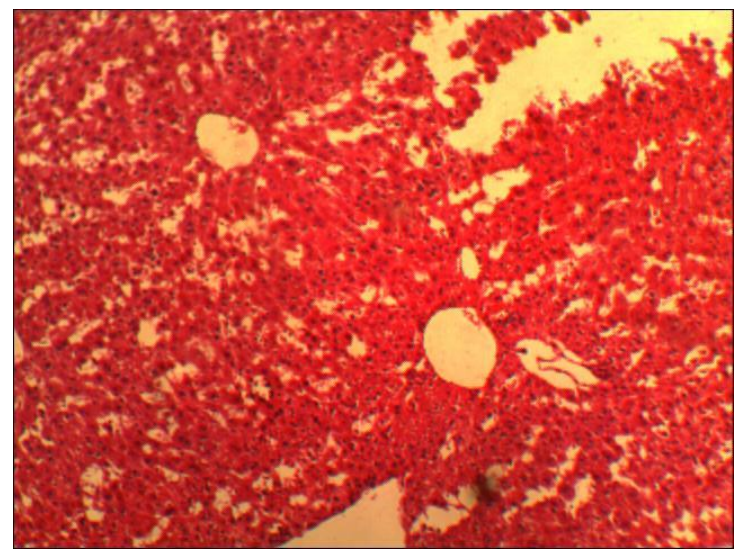

KIDNEY

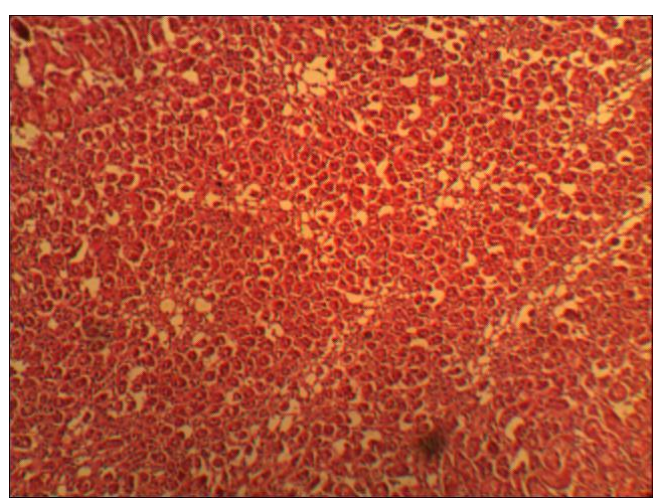

US irradiated Kidney

Figure 1 Microscopic photos showing morphological details of study groups for liver and kidney tissue samples, A: Control samples, and B: US irradiated samples. 


\section{$\underline{\text { Hematological studies }}$}

CBC parameters for sample groups were tabulated in Table 1(Mean \pm SE). The results are still within normal ranges. There was no significant difference $(\mathrm{P}<0.01)$ in values of hemoglobin $(\mathrm{HGB})$, red blood cells $(\mathrm{RBCs})$, and other red blood indices such as haematocrit (HCT), mean corpuscular volume (MCV), mean corpuscular heamoglobin content (MCHC) between research groups Table 1.

\begin{tabular}{|c|c|c|c|c|c|c|c|c|}
\hline & $\begin{array}{l}\text { RBC } \\
(M / \mu L)\end{array}$ & $\begin{array}{l}\text { HGB } \\
\text { (g/dL) }\end{array}$ & $\begin{array}{l}\text { HCT } \\
(\%)\end{array}$ & $\begin{array}{l}\text { MCV } \\
\text { (fL) }\end{array}$ & $\begin{array}{l}\text { MCH } \\
\text { (pg) }\end{array}$ & $\begin{array}{l}\text { MCHC } \\
(\mathrm{g} / \mathrm{dL})\end{array}$ & $\begin{array}{l}\text { PLT } \\
(\mathbf{m m} / \mu \mathrm{L})\end{array}$ & $\begin{array}{l}\mathrm{WBC} \\
(\mathrm{mm} / \mu \mathrm{L})\end{array}$ \\
\hline Control & $11.4 \pm 1.8$ & $14 \pm 1.2$ & $44.6 \pm 2.4$ & $59.4 \pm 2.5$ & $18 \pm 1$ & $38 \pm 3.2$ & 671 & 1.4 \\
\hline US pulsed & $12 \pm 0.6$ & $15 \pm 1.2$ & $46 \pm 2.4$ & $61 \pm 2.5$ & $20 \pm 2$ & $40 \pm 2.2$ & 559 & 1.5 \\
\hline $\begin{array}{l}\text { US } \\
\text { continuous }\end{array}$ & $9.8 \pm 1.6$ & $14 \pm 1.2$ & $51.6 \pm 2.4$ & $66.4 \pm 2.5$ & $18 \pm 1$ & $38 \pm 1$ & 633 & 1.1 \\
\hline
\end{tabular}

\section{Total Antioxidant activity}

The lipid peroxidation activity and malonaldehyde levels are shown in Table2. According to our study, US irradiated groups exhibited significantly high levels of MDA, as compared with the control group. Regarding the antioxidant capacity, all study groups showed decreased activities of antioxidants (SOD, CAT, GR, GST, and TAC) in comparison with normal animals(p>0.05). Table2.

F: F value for ANOVA test, a: Significant with Normal group*: Statistically significant at $p \leq 0.05$ Data was expressed by using mean \pm SD.

\section{Effects on biochemical parameters}

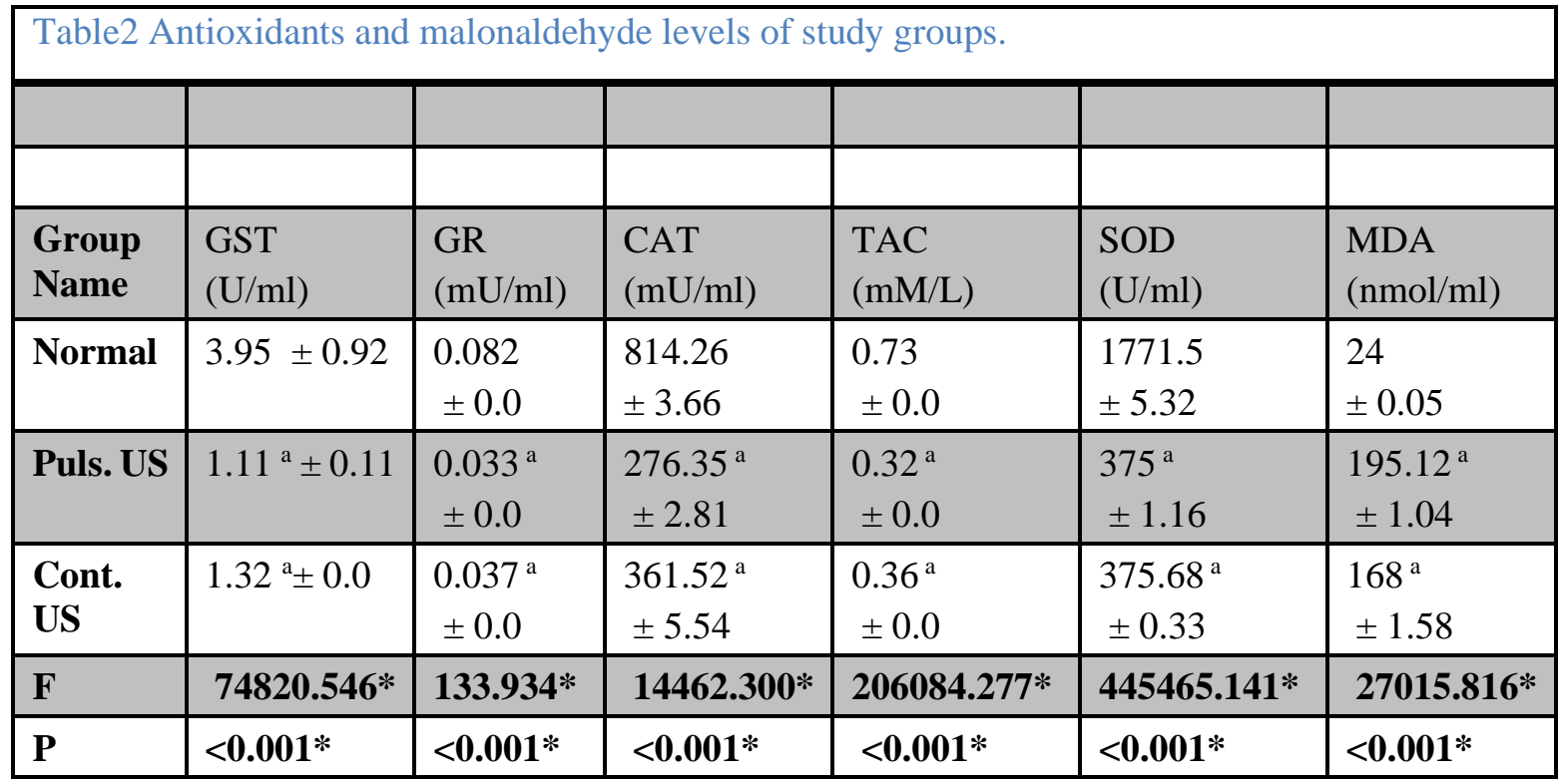

enzymes (ALT, AST) and kidney function parameters (urea and creatinine) are represented in there is no significant alteration in study groups compared with a control group. 

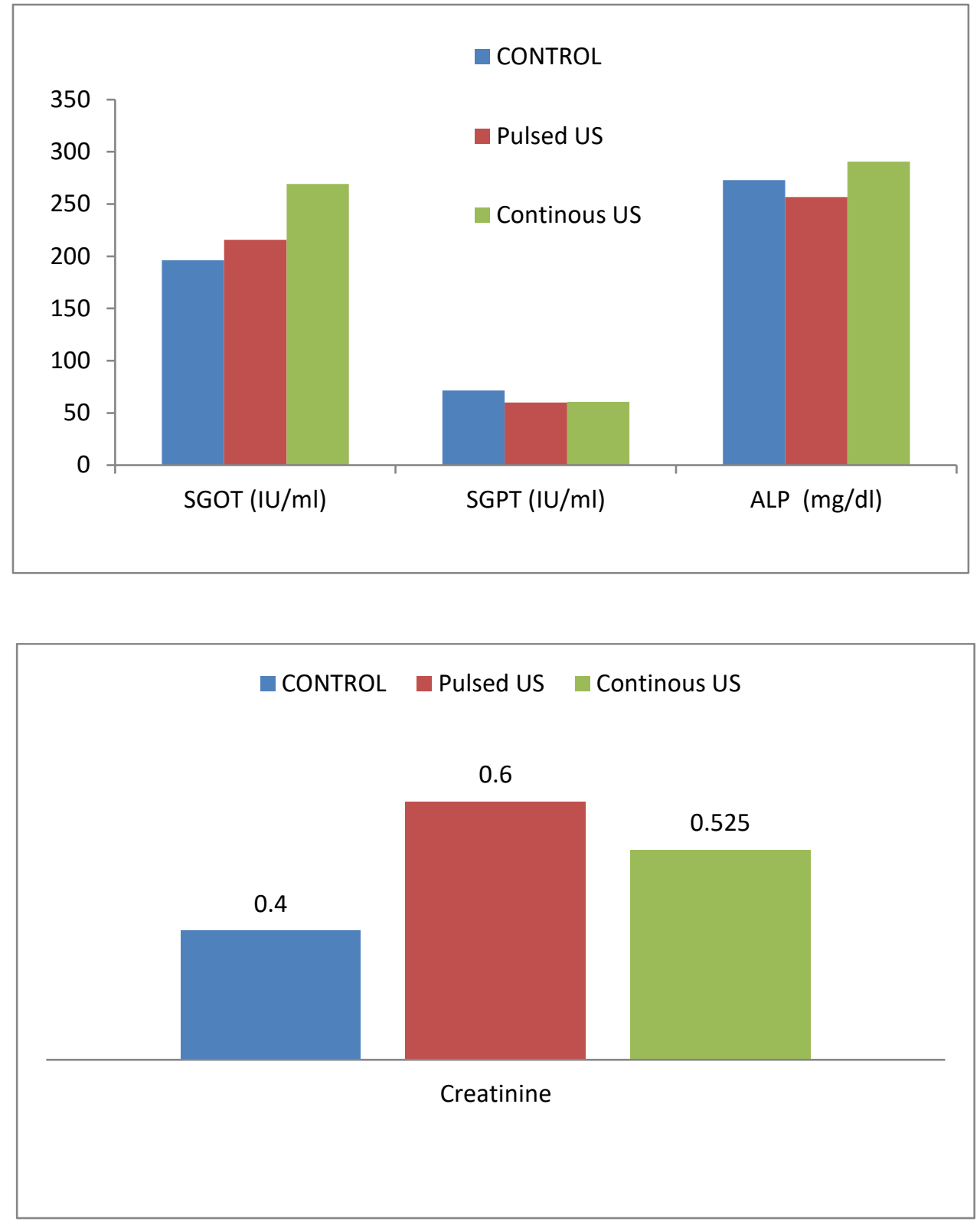

\section{Discussion}

Upgrading technology makes our present world a pool of electromagnetic waves irradiation1.our daily lifestyle aids in increase the rate of exposure including industrial noising, working environment, cell phones, Home-aid tools, microwave, oven, and electric heater and computers.

In the current study, the effect of therapeutic ultrasound waves irradiation on the liver and kidney function of male albino mice was investigated. The outcome results showed a significant no alteration at the hematological and biochemical levels (liver enzymes and kidney function tests) in comparison with a nonirradiated control group. Histopathological studies revealed no obvious change in cellular ultrastructure of mice liver and kidney cells in comparison with control groups $^{12}$.

Monitoring the oxidative stress parameters, lipid peroxidation indication was evaluated by MDA level, irradiated groups with I.R.L. or U.S. exhibited significantly high levels of MDA, as compared with the control group. This finding was 
coincident with the work of Luis DT ET al and Errki J V. $(4,13,12)$. The antioxidant capacity was estimated as a preventive line for liver and kidney injury. Enzymatic (like SOD), and nonenzymatic antioxidants (such as GST) showed decreased activities in comparison with a control group.

In summary, from our finding on irradiated male albino mice, we can occlude that using therapeutic US irradiation with output power from 0.5 $3 \mathrm{~W} / \mathrm{cm} 2$ and pulsed mode (pulse frequency 1000 $\mathrm{Hz}$, duty ratio $1 / 3$ and average power density from $0.15-1 \mathrm{~W} / \mathrm{cm}^{2}$ ) can be safely used for the

\section{References:}

1 Bożena Smagowska. Effects of Ultrasonic Noise on the Human Body.International Journal of Occupational Safety and Ergonomics (JOSE) 2013, Vol. 19, No. 2, 195-202.

2 R. Benson, R. Madan, R. Kilambi b, S. Chander. Radiation induced liver disease: A clinical update.Journal of the Egyptian National Cancer Institute (2016) 28, 7-11.

3 Tai A, Erickson B, Li XA. Extrapolation of normal tissue complication probability for different fractionations in liver irradiation.Int $\mathbf{J}$ RadiatOncolBiol Phys 2009;74:283-9.

4 Ter Haar G. Ultrasonic imaging: safety considerations.Interface Focus. 2011 Aug 6;1(4):686-97. doi: 10.1098/rsfs.2011.0029.

5 Paul Okunieff, Steven Swarts, Peter Keng, Weimin Sun, Wei Wang, Jung Kim, Shanmin Yang, Hengshan Zhang, Chaomei Liu, Jacqueline P. Williams, Amy K. Huser, and Lurong Zhang antioxidants reduce consequences of radiationexposure.Adv Exp Med Biol. 2008;614:165-78. doi: 10.1007/978-0-387-749112_20.

6 Sha Li, Hor-Yue Tan, Ning Wang, Zhang-Jin Zhang, Lixing Lao, Chi-Woon Wong and YibinFeng .The Role of Oxidative Stress and Antioxidants inLiver Diseases.Int J Mol Sci. 2015 Nov 2;16(11):26087-124. doi: 10.3390/ijms 161125942 .

7 Tomohito Sato, Manabu Kinoshita, Tetsuo Yamamoto, MasatakaIto,Takafumi Nishida, therapeutic purpose with no significant alteration in cell integrity of both liver and kidney of male albino mice.

\section{Acknowledgement}

I wish to acknowledge the help provided by $D r$. Eslam A A Nasser, PhD laboratory specialist at Alexandria fever hospital, in sample management and animal care Laboratory. Assistance provided by Dr. Ahmed A. Yameny, head of the society of pathological biochemistry and hematology, chief of medical laboratory specialists syndicate.

MasaruTakeuchi, DaizohSaitoh, Shuhji Seki, YasuoMukai.Treatment of IrradiatedMice with High-DoseAscorbic Acid Reduced Lethality.PLoS One. 2015 Feb 4;10(2):e0117020. doi: 10.1371/journal.pone.0117020.

8 Deepa D'souza, Babu G Subhas, Shishir Ram Shetty, Preethi Balan. Estimation of serum malondialdehyde in potential ly malignant disorders and post antioxidant treated patients: A biochemical study. Contemp Clin Dent. 2012 Oct-Dec; 3(4): 448451. doi: 10.4103/0976-237X.107438.

9 M Salahuddin, H Akhter, S Akter, MA Miah, and $\mathrm{N}$ Ahmad.Effects of probiotics on haematology and biochemical parameters in mice.The Bangladesh Veterinarian (2013) 30(1) : 20 - 24 diagnostics.com/.../Alanine_Aminotransferase_(A LT)

11 www.spectrumdiagnostics.com/data/Creatinine_(colorimetric).pd $\mathrm{f}$

12 Erkki J. Valtonen .Influence of Ultrasonic Radiation in the Medical Therapeutic Range on the Fine Structure of the Liver Parenchymal Cell.VALTONEN, E. J., 1967.

13 Sulbha K. Sharma, Gitika B. Kharkwal,Mari Sajo, MS, Ying-Ying Huang, Luis De Taboada, FSEE, Thomas McCarthy, DVM, and Michael R. Hamblin.Dose Response Effects of $810 \mathrm{~nm}$ Laser Light on Mouse PrimaryCortical Neurons.Lasers Surg Med. 2011 Sep;43(8):851-9. doi: 10.1002/lsm.21100. 